\title{
Representaciones Sociales de la Obesidad en Jóvenes Preuniversitarios y Universitarios
}

\author{
Social Representations of Obesity in Pre-University and University Youth \\ Erika Collipal L..* \& María Pía Godoy B.**
}

COLLIPAL, L. E. \& GODOY, B. M. P. Representaciones sociales de la obesidad en jóvenes preuniversitarios y universitarios de la ciudad de Temuco. Int. J. Morphol., 33(3):877-882, 2015.

RESUMEN: La obesidad es considerada actualmente una epidemia mundial, en niños, adolescentes y adultos. Estudios clínicos en niños obesos han demostrado que tienen riesgo de variadas enfermedades asociadas, como enfermedades psicológicas, ortopédicas por sobrecarga para el aparato locomotor, disnea de esfuerzo ante el ejercicio, factores de riesgo cardiovasculares, alteraciones endocrinas y alteraciones hepáticas. Por lo tanto es muy relevante la prevención de la obesidad en la infancia y la adolescencia, y conocer los significados compartidos que estos grupos presentan sobre la obesidad, permite un acercamiento a la problemática. Con el objetivo de determinar el significado que tiene para los adolescentes preuniversitarios y universitarios del área de la salud de la Universidad de La Frontera, Temuco, Chile, y con la convicción que estos estudiantes serán los profesionales que tendrán participación en educación en salud, es que realizamos este estudio de corte transversal, utilizando para la entrevista la Técnica de las Redes Semántica Naturales. Se seleccionaron a través de un muestreo por conglomerado 200 adolescentes de ambos sexos entre 18 a 20 años, estudiantes del Preuniversitario, de las carreras de Terapia Ocupacional, Enfermería y Nutrición y Dietética. Los principales resultados señalan que para los estudiantes los términos con alto valor semántico del concepto Obesidad son: Enfermedad, comida, grasa, problema y sedentarismo. Estos resultados demuestran que los estudiantes reconocen que la obesidad es una enfermedad, que el sedentarismo y la alimentación son conceptos con un alto valor semántico asociado a la obesidad, por lo tanto en la educación de los niños se debe estimular la actividad física, la buena alimentación y que debería haber un manejo integral del problema con especial intención en la familia y en los establecimientos educacionales.

PALABRAS CLAVE: Obesidad; Educación; Adolescentes; Redes semánticas; Sedentarismo.

\section{INTRODUCCIÓN}

La prevención de la obesidad en la infancia y la adolescencia presenta una gran relevancia, debido a que son edades críticas en la que los hábitos alimenticios, la construcción personal y la práctica de actividad física está en proceso de consolidación, por lo tanto tienen más probabilidades de modificarse a través de la educación. La población actual ha adquirido una nueva cultura alimentaria en la que incorpora alimentos y comida de alto contenido energético, menor consumo de frutas y verduras, además la vida tiende a ser cada vez más sedentaria con toda la tecnología que está a disposición de los adolescentes, lo que disminuye el gasto energético

El sedentarismo es un problema muy grave en nuestra población, las encuestas de los últimos años de Calidad de Vida y Salud (MINSAL, 2006, 2010) muestran que un
$24,1 \%$ pasa la mayor parte del tiempo sentado (a) y camina poco, un $72,8 \%$ no practica deportes en el mes. En relación a la edad un $22,3 \%$ de la población de 15 a 19 años pasa la mayor parte del tiempo sentado, un $89,2 \%$ no practica deporte o no realiza actividad física fuera de su horario de trabajo durante 30 minutos o más cada vez.

La encuesta nacional de salud 2009-2010 indica que la prevalencia de sobre peso en los hombres es de $45,3 \%$ y en las mujeres de $33,6 \%$, la obesidad en hombres es de un $19,2 \%$ y en mujeres de $30,7 \%$. Según nivel educacional en relación a la obesidad es de 35,5\% en la población con bajo nivel educacional esto es menos de 8 años de estudio, un $24,7 \%$ de 8 a 12 años de estudio y con más de doce años de un 18,5\%. Diversos investigadores (Andersen, 2000; Ebbeling et al., 2002; Boon \& Clydesdale, 2005; Veugelers

\footnotetext{
* Facultad de Medicina, Universidad de La Frontera, Temuco, Chile.

${ }^{* *}$ Facultad de Educación, Universidad de La Frontera, Temuco, Chile.
} 
\& Fitzgerald, 2005) indican que la obesidad y el sobrepeso se han convertido en un problema de salud pública en los países industrializados. La obesidad constituye en la actualidad la enfermedad nutricional más prevalente en la población menor de 20 años (WHO, 2003).

Carrasco Dell'Aquila et al. (2009) en este mismo sentido señalan que la prevalencia de obesidad en adolescentes en Chile ha aumentado en el orden del 1,5\% por año, además plantean que países como USA y Japón presentan cifras similares.

En relación a la actividad física de los adolescentes, actividades tales como ver TV, jugar nintendo y uso del computador, ocupan gran parte del tiempo libre, determinando un gasto calórico mínimo, por lo que se les ha considerado como horas de inactividad física (Burrows, 2000; Olivares et al., 1999), ocupando un porcentaje importante del día de muchos niños y adolescentes. Kain et al. (2001) manifiestan que las niñas gastan más tiempo en actividades sedentarias que los niños. Collipal et al. (2006) indica que las adolescentes relacionan la obesidad con el sedentarismo, no siendo un factor que tiene mucha relación con el termino obesidad.

Un estudio realizado por Skidmore \& Yarnell (2004) indica que entre el 20 y 25\% de los adultos de Gran Bretaña son obesos, de acuerdo con el criterio de la OMS (IMC mayor o igual a $30 \mathrm{Kg} / \mathrm{m} 2$ ), y que muchos estudios epidemiológicos sugieren que la actividad física en el trabajo, escuela y la recreación han declinado a niveles mínimos y los comportamientos sedentarios, tales como, ver televisión y juegos por computador, han llegado a ser los mayores pasatiempos. Por lo tanto los requerimientos energéticos son mucho menor en las generaciones recientes, esto obliga a incrementar las clases de educación física en escolares por lo menos a dos por semana y ensayar diferentes protocolos de intervención que permitan reducir o prevenir el problema en niños y jóvenes.

La percepción del propio estado nutricional en Kain et al., indica que el 50\% de los niños con sobrepeso y el $48 \%$ con obesidad se autocalifica como tal, en tanto solo el $36 \%$ de las niñas con sobrepeso y $38 \%$ de las que presentan obesidad, se definió en la categoría real. El resto se considera de peso normal.

El vínculo que una persona posee con su propio cuerpo, ya sea, éste de unión o desvinculación, depende estrechamente del contexto relacional e intersubjetivo, es decir, de la relación que se establece entre el adolescente y el sistema de cuidado y contexto que lo rodea. Resaltando las experiencias tempranas, que posteriormente se convierten en los estados subjetivos en relación a la percepción corporal del individuo (Carrasco Dell'Aquila et al.).
Considerando la relación de la obesidad con el sedentarismo y la relación con el tiempo destinado a ver televisión, según Olivares et al. (1999) el 40,7\% de los niños veía entre 1 a 3 horas diarias y el 20,2\% más de tres horas durante los días que asistían al colegio. Según Olivares etal. (2003) el 56,5\% de los niños veía más de 3 horas diarias de TV los días de colegio, cifra que aumentó a 58,8\% el día sábado. Gattas et al. (1996) reportaron que los escolares chilenos de primero a cuarto básico de la región Metropolitana, veían 3 horas diarias de TV en el caso de los varones y 2,4 horas en las mujeres durante los días de semana, cifra que aumentó a 4,4 y 3,4 h durante los fines de semana. El número de horas diarias que los niños ven televisión es especialmente relevante por su asociación con la falta de actividad física, la que a su vez se asocia a obesidad (Dietz \& Gortmaker, 1985; Klesges et al., 1993; Taras et al., 1989).

En la población escolar chilena, la tendencia muestra que la obesidad se ha casi triplicado en varones y mujeres, al comparar estudios realizados en la década del 80 y del 90 (Muzzo et al., 1999). Jadue et al. (1999) al analizar la obesidad según nivel socioeconómico, encontró una prevalencia de $28,9 \%$ en mujeres y $18,6 \%$ en hombres de nivel socioeconómico bajo. Más del $90 \%$ de las mujeres no hacen ejercicio en el tiempo libre y en los hombres este problema aumenta con la edad.

Silva et al. (2008), señalan que el control de la obesidad en los colegios debe ser prioridad, es necesario crear conciencia en los padres, profesores y en todo el ámbito educativo para instaurar normas sobre el control de la obesidad en los colegios ya que esta enfermedad se instaura en los primeros años de vida. Vásquez et al. (2005) alertan sobre la necesidad de educar nutricionalmente a los padres y comunidad educativa, así como diseñar un currículo educacional para preescolares que fomente el hábito de la alimentación saludable y la actividad física continua.

En estudiantes de enseñanza media de la región metropolitana en un estudio realizado por Burrows et al. (2008) en establecimientos de enseñanza municipal, colegios subvencionados y colegios particulares en relación a la actividad física programada indica que el $68,2 \%$ de las mujeres y $34,3 \%$ de los varones de colegios municipales tenían menos de $2 \mathrm{~h}$ vs. $5,5 \%$ y $6,8 \%$ de las mujeres y varones respectivamente de colegios privados. Por otra parte, $47,3 \%$ y $57,0 \%$ de mujeres y varones, respectivamente de colegios privados tenían 4 h o más de ejercicio programado versus $13,4 \%$ y $41,2 \%$ respectivamente, en los colegios municipales.

En escolares de edades comprendidas entre los 10 a 12 años, para Guerra Cabrera et al. (2009) según horas 
de actividad física diaria, el 38,0\% de los que admitieron no realizar ninguna actividad física fueron casos sobrepeso u obeso frente al 14,0\% de los controles. Esta diferencia tan profunda a favor de la inactividad fue muy significativa, con un riesgo 5 veces mayor de presentar exceso de peso. Al analizar las horas de actividad física según grupos se obtuvo que el $86 \%$ de los escolares normopeso tuvo más de una hora diaria de ejercicios, mientras que los sobrepeso solo el $62 \%$ realizó alguna actividad.

En relación a la actividad física para prevenir la obesidad, Salinas \& Vio (2003) elaboraron una guía de vida activa cuyos mensajes simples son: caminar a lo menos 30 minutos diarios; realizar ejercicios de estiramiento; recrearse, ojala de manera activa, haciendo ejercicio; realizar pausas activas en el trabajo; realizar como ideal 30 min de ejercicio físico más intenso por lo menos tres veces a la semana y adecuar su intensidad al ritmo personal. La actividad física es un componente clave del balance energético en niños y adolescentes, considerado como uno de los factores que promueven un óptimo crecimiento y desarrollo, así como también una vida más saludable (Torun et al., 1996).

Sin embargo persisten interrogantes de cuál es el significado de la obesidad para los adolescentes, atendiendo a esta interrogante es que hemos realizado esta investigación para determinar cuál es el significado de la obesidad en adolescentes preuniversitarios y universitarios de la Facultad de Medicina de la Universidad de La Frontera y de esta forma poder realizar actividades que tiendan a educar para disminuir los índices de sobrepeso y obesidad.

\section{MATERIAL Y MÉTODO}

Para obtener la representación del concepto obesidad, desde la perspectiva de los estudiantes, aplicamos la técnica de Redes Semánticas Naturales, basada en los estudios de Valdes Medina (1998). La muestra estuvo constituida por 200 estudiantes cuyas edades fluctuaban entre 18 a 22 años del Preuniversitario y de la carrera de Terapia Ocupacional, Enfermería y Nutrición y Dietética de la Facultad de Medicina Universidad de La Frontera, Temuco, Chile. Los estudiantes encuestados firmaron un consentimiento donde se les informo que la encuesta era individual, anónima y se les explicaba el propósito de la investigación.

La técnica consistió en entregar una hoja impresa con la palabra estímulo "obesidad", acompañada de dos columnas en las cuales una correspondía a la palabra definidora y la otra columna a la jerarquía. Se les solicitó anotar en la primera columna un mínimo de diez palabras que definieran al estímulo y en la segunda que jerarquizaran las palabras que dieron como definidoras, de acuerdo a la importancia, relación o cercanía con la palabra estímulo presentado.

Se analizaron los siguientes resultados entregados por la técnica, Valor J: corresponde al total de palabras definidoras, indicador de la riqueza semántica de la red. Valor M: Resultado de la multiplicación de la frecuencia de aparición por la jerarquía obtenida para cada una de las palabras. Conjunto SAM: grupo de diez palabras definidoras que obtuvieron los mayores valores $M$ totales. Valor FMG: indicador en términos de porcentaje de la distancia semántica que hay entre las diferentes palabras definidoras del conjunto SAM.

\section{RESULTADOS}

El resultado del análisis de la representación que tienen los adolescentes de la obesidad se obtuvo de las palabras generadas por los sujetos.

Los resultados de la multiplicación de la frecuencia de aparición por la jerarquía obtenida para cada una de las palabras y el indicador en términos de porcentaje de la distancia semántica que hay entre las diferentes palabras definidoras del conjunto SAM para estudiantes del preuniversitario, de Terapia Ocupacional, Nutrición y Enfermería, se observan en las Tablas I, II, III y IV, respectivamente.

Tabla I. Valor M y FMG para conjunto SAM. Estudiantes del preuniversitario.

\begin{tabular}{lcc}
\hline Conjunto SAM & Valor M & Valor FMG \\
\hline Enfermedad/Trastorno & 243 & 100 \\
Gordura/Gordo & 173 & 71 \\
Sedentarismo & 162 & 67 \\
Sobrepeso & 98 & 40,3 \\
Grasa & 92 & 37,8 \\
Chatarra & 80 & 33 \\
Guata/Guatón/Panzón & 77 & 31,6 \\
Comida & 72 & 29,6 \\
Exceso & 68 & 27,9 \\
Flojo & 50 & 20,5 \\
Rollos & 41 & 16,8 \\
Ancho & 37 & 15,2 \\
Peso & 37 & 15,2 \\
Cansancio & 34 & 13,9 \\
Diabetes & 32 & 13,1 \\
\hline
\end{tabular}


Tabla II. Valor M y FMG para conjunto SAM. Estudiantes de Terapia Ocupacional.

\begin{tabular}{lcc}
\hline Conjunto SAM & Valor M & Valor FMG \% \\
\hline Enfermedad & 272 & 100 \\
Comida/Alimentación & 167 & 61,3 \\
Grasas & 146 & 53,6 \\
Sedentarismo & 132 & 48,8 \\
Gordura & 123 & 45,2 \\
Exceso & 116 & 42.6 \\
Sobrepeso & 78 & 28,6 \\
Ansied ad & 44 & 16,1 \\
Chatarra & 39 & 14,3 \\
Depresión & 38 & 13,9 \\
Cansancio & 36 & 13,2 \\
Adipositos & 30 & 11 \\
Anorexia & 29 & 10,6 \\
Malnutrición & 28 & 10,2 \\
Trastorno & 23 & 8,4 \\
\hline
\end{tabular}

Tabla III. Valor M y FMG para conjunto SAM. Estudiantes de Nutrición.

\begin{tabular}{lcc}
\hline Conjunto SAM & Valor M & Valor FMG \% \\
\hline Enfermedad & 275 & 100 \\
Sedentarismo & 165 & 60 \\
Comida & 164 & 60 \\
Grasa & 162 & 58,9 \\
Exceso & 160 & 58,2 \\
Sobrepeso & 98 & 35,6 \\
Chatarra & 70 & 25,5 \\
Ansied ad & 59 & 21,5 \\
Depresión & 48 & 17,5 \\
Mal Nutrición & 34 & 12,4 \\
Dieta & 32 & 11,6 \\
Muerte & 30 & 10,9 \\
Colesterol & 29 & 10,5 \\
Cansancio & 27 & 9,8 \\
Problema & 27 & 9,8 \\
\hline
\end{tabular}

\section{DISCUSION}

La obesidad es una enfermedad que afecta la vida social no solo de los adolescentes si no de todas las personas que la padecen, los estudiantes encuestados lo manifiestan a través de representaciones como ansiedad, cansancio, depresión, vergüenza, trastorno, discriminación, bullying, todo en el contexto de ciento tres términos incluidos en el valor J de la Red Semántica.

Incluyen en el valor $\mathrm{J}$ la comida chatarra, grasas, dulces, concordando con Olivares et al. (1999, 2003), quienes indican que la primera prioridad de compra de los niños son productos dulces y salados que adquieren en los quios-
Tabla IV. Valor M y FMG para conjunto SAM. Estudiantes de Enfermería.

\begin{tabular}{lcc}
\hline Conjunto SAM & Valor M & Valor FMG \% \\
\hline Enfermedad & 345 & 100 \\
Comida & 193 & 55,9 \\
Problema & 184 & 53,3 \\
Gordura & 157 & 45,5 \\
Exceso & 143 & 41,4 \\
Grasa & 127 & 36,8 \\
Sobrepeso & 119 & 34,4 \\
Ansied ad & 116 & 33,6 \\
Insano & 97 & 28,1 \\
Sedentarismo & 74 & 21,4 \\
Inseguridad & 46 & 13,3 \\
Nutrición & 37 & 10,7 \\
Dieta & 35 & 10,1 \\
Chatarra & 34 & 9,8 \\
Descontrol & 33 & 9,5 \\
\hline
\end{tabular}

cos del colegio y los comerciales favoritos en la publicidad televisiva son productos envasados dulces y salados.

Pero del mismo modo, los estudiantes consultados reconocen a la obesidad como una enfermedad (Fig. 1) y hacen referencia en general a causales como la ingesta inadecuada de comida y el sedentarismo, flojera, cansancio, peso, inactividad, agotamiento. En escolares de edades comprendidas entre los 10 a 12 años, en un estudio de Guerra Cabrera et al. según horas de actividad física diaria, el 38,0\% de los que admitieron no realizar ninguna actividad física fueron casos sobrepeso u obeso frente al 14,0\% de los controles. Esta diferencia tan profunda a favor de la inactividad fue muy significativa, con un riesgo 5 veces mayor de presentar exceso de peso. Al analizar las horas de actividad física según grupos se obtuvo que el $86 \%$ de los escolares normopeso tuviera más de una hora diaria de ejercicios, mientras que los sobrepeso solo el $62 \%$ realizó alguna actividad. Es importante esta información para poder estimular e intensificar acciones que lleven a mantener un estilo de vida saludable y de esta forma contribuir a disminuir los índices de sobrepeso y obesidad.

Por otro lado los estudiantes destacan como representaciones de la obesidad conceptos asociados a consecuencias sociales en las personas con obesidad, mencionando algunos como ansiedad, depresión, vergüenza y discriminación.

Es unánime la percepción general que uno de los principales focos de prevención de la obesidad es desde edades tempranas y debe ser una educación contextual. En este sentido señala Vásquez et al. a la escuela y al hogar como los ejes claves de educación para el logro de estilos de vida sa- 


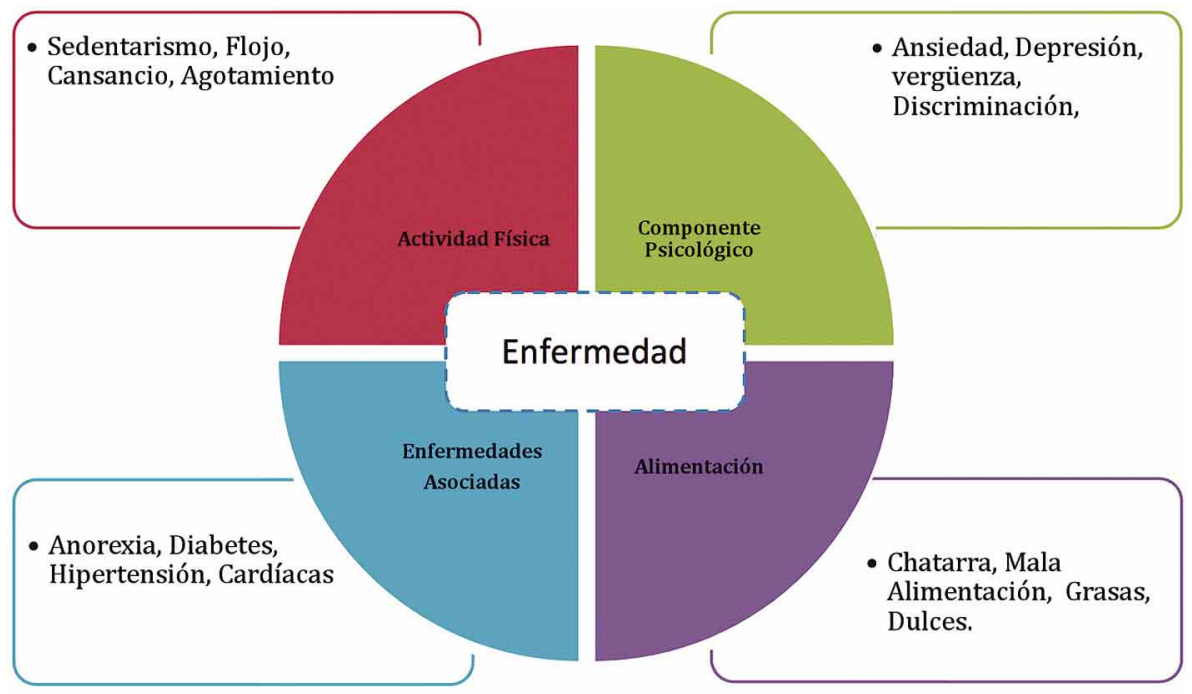

Fig. 1 Representación Resultados Redes. ludables; del mismo modo Carrasco Dell'Aquila et al., resaltan que la relación de un individuo con su propio cuerpo, se ve influenciado por el sistema de cuidado y contexto que lo rodea, en especial durante las experiencias tempranas que posteriormente impactan en su vida.

Por ende la prevención de la obesidad como el conocimiento de las representaciones que los adolescentes poseen de ésta, permite avanzar en la comprensión para la elaboración de formas de educación que promuevan el desarrollo integral de las personas, en especial en ésta temática que posee una connotación social, no sólo por el aumento de la obesidad, sino también porque es una enfermedad que presenta impactos en otras áreas de la vida de las personas, en especial en el auto concepto, autoestima y la relación con el propio cuerpo, así como impacta también en la relación con los otros.

COLLIPAL, L. E. \& GODOY, B. M. P. Social representations of obesity in pre university and university youth from the city of Temuco. Int. J. Morphol., 33(3):877-882, 2015.

SUMMARY: Obesity is currently considered a global epidemic in children, adolescents and adults. Clinical studies have shown obese children having various associated diseases such as psychological diseases, orthopedic problems due to overload on the musculoskeletal system, dyspnea during exercise, cardiovascular risk factors, endocrine disorders and liver disorders. The prevention of obesity in childhood and adolescence is very relevant, and knowing the information shared by these groups on obesity, is a way to better approach the issue. This cross sectional study was carried out in order to determine the concept preuniversity and undergraduate university students who are in the health care areas have. Furthermore, it is based on the fact that these students will be the professionals who will be active participants in health education. The students were selected by cluster sampling: 200 adolescents of both sexes between 18-20 years of age of pre-university students of the occupational therapy, nursing and nutrition, and dietetics degree programs. The main results indicate that students' concepts in terms with semantic value of obesity are as follows: disease, fatty foods, problems and a sedentary lifestyle. These results demonstrate that students acknowledge that obesity is an illness, in which sedentary lifestyle and nutrition have a high semantic value associated with obesity. Child education should encourage physical activity and healthy nutritional habits, while management of the problem should be all-inclusive and place special emphasis on the family and educational institutions.

KEY WORDS: Obesity; Education; Teens; Semantic networks; Sedentary.

\section{REFERENCIAS BIBLIOGRÁFICAS}

Andersen, R. E. The spread of the childhood obesity epidemic. $C$. M. A. J., 163(11):1461-2, 2000.

Burrows, A. R. Prevención y tratamiento de la obesidad desde la niñez: la estrategia para disminuir las enfermedades crónicas no transmisibles del adulto. Rev. Med. Chile, 128(1):105-10, 2000.

Burrows, A. R.; Díaz, B. E.; Vito Sciaraffia, M.; Gattas, Z. V.; Montoya, C. A. \& Lera, M. L. Hábitos de ingesta y actividad física en escolares, según tipo de establecimiento al que asisten. Rev. Med. Chile, 136(1):53-63, 2008.

Boon, C. S. \& Clydesdale, F. M. A Review of childhood and adolescent obesity interventions. Crit. Rev. Food Sci. Nutr., 45(7-8):511-25, 2005. 
Carrasco Dell'Aquila, D.; Gómez Castro, E. \& Staforelli Mosca, A. Obesidad y Adolescencia: Exploración de Aspectos Relacionales y Emocionales. Ter. Psicol., 27(1):143-9, 2009.

Collipal, E.; Silva, H.; Vargas, R \& Martínez, C. The meaning of obesity for adolescents of Temuco-Chile. Int. J. Morphol., 24(2):259-62, 2006.

Dietz, W. H. Jr. \& Gortmaker, S. L. Do we fatten our children at the television set? Obesity and television viewing in children and adolescents. Pediatrics, 75(5):807-12, 1985.

Ebbeling, C. B.; Pawlak, D. B. \& Ludwig, D. S. Childhood obesity: public-health crisis, common sense cure. Lancet, 360(9331):473-82, 2002.

Gattás, Z. V.; Barrera, A. G.; Riumalló, S. J \& Uauy, D. R. Actividad física en escolares chilenos normales y de baja talla. Rev. Chil. Pediatr., 67(5):212-8, 1996.

Guerra Cabrera, C. E.; Vila Díaz, J.; Apolinaire Pennini, J. J.; Cabrera Romero, A. C.; Santana Carballosa, I. \& Almaguer Sabina, P. M. Factores de riesgo asociados a sobrepeso y obesidad en adolescentes. MediSur, 7(2):25-34, 2009.

Jadue H., L.; Vega M., J.; Escobar S., M. C.; Delgado B., I.; Garrido G., C.; Lastra M., P.; Espejo E., F \& Peruga U, A. Factores de riesgo para las enfermedades no transmisibles: Metodología y resultados globales de la encuesta de base del programa CARMEN (Conjunto de Acciones para la Reducción Multifactorial de las Enfermedades no Transmisibles). Rev. Med. Chile, 127(8):1004-13, 1999.

Kain B., J.; Olivares C., S.; Castillo A., M. \& Vio D., F. Validación y aplicación de instrumentos para evaluar intervenciones educativas en obesidad de escolares. Rev. Chil. Pediatr., 72(4):308$18,2001$.

Klesges, R. C.; Shelton, M. L. \& Klesges, L. M. Effects of television on metabolic rate: potential implications for childhood obesity. Pediatrics, 91(2):281-6, 1993.

Ministerio de Salud (MINSAL). II Encuesta de Calidad de Vida y Salud Chile 2006. Informe de Resultados Total Nacional. Santiago, Ministerio de Salud, Subsecretaria de Salud Publica, División Planificación Sanitaria, Gobierno de Chile, 2006.

Ministerio de Salud (MINSAL). Encuesta Nacional de Salud. Santiago, MINSAL, Gobierno de Chile, 2010.

Muzzo, S.; Cordero, J. \& Burrows, R. Cambios en la prevalencia del exceso de peso del escolar chileno en los últimos 8 años. Rev. Chil. Nutr., 26(3):311-5, 1999.

Olivares, S.; Albala, C.; García, F \& Jofré, I. Publicidad televisiva y preferencias alimentarias en escolares de la región metropolitana. Rev. Med. Chile, 127:791-9, 1999.
Olivares, S.; Yáñez, R \& Díaz, N. Publicidad de alimentos y conductas alimentarias en escolares de $5^{\circ}$ a $8^{\circ}$ básico. Rev. Chil. Nutr., 30(1):36-42, 2003.

Silva M., H.; Collipal L., E.; Martínez, C \& Torres, I. Analysis of BMI and somatotype in a sample of adolescents were overweight and obesity in Temuco - Chile. Int. J. Morphol., 26(3):707-11, 2008.

Skidmore, P. M. \& Yarnell, J. W. The obesity epidemic: prospects for prevention. Q. J. M., 97(12):817-25, 2004.

Taras, H. L.; Sallis, J. F.; Paterson, T. L., Nader, P. R. \& Nelson, J. A. Television's influence on children's diet and physical activity. J. Dev. Behav. Pediatr., 10(4):176-80, 1989.

Torun, B.; Davies, P.; Livingstone, M. B.; Paolisso, M.; Sackett, R \& Spurr, G. B. Energy requirements and dietary energy recommendations for children and adolescents 1 to 18 years old. Eur. J. Clin. Nutr., 50(Suppl. 1):S37-80, 1996.

Valdes Medina, J. L. Las redes semánticas naturales: usos y aplicaciones en psicología social. $2^{\mathrm{a}}$ ed. Ciudad de México, Universidad Autónoma del Estado de México, 1998.

Vásquez, V. F.; Cardona, H. O.; Andrade, S. M \& Salazar, R. G. Balance de energía, composición corporal y actividad física en preescolares eutróficos y obesos. Rev. Chil. Pediatr., 76(3):266-74, 2005.

Salinas, J. \& Vio, F. Guías de Vida Activa para la Población Chilena. Santiago de Chile, Ed. Andros, 2003.

Veugelers, P. J. \& Fitzgerald, A. L. Prevalence of and risk factors for childhood overweight and obesity. C. M. A. J., 173(6):60713, 2005.

World Health Organization (WHO). Diet, nutrition and the prevention of chronic diseases. Report of a joint WHO/FAO Expert Consultation. Geneva, WHO Technical report series 916, 2003.

Dirección para Correspondencia:

Erika Collipal L.

Unidad de Anatomía

Facultad de Medicina

Universidad de la Frontera

Temuco

CHILE

Email: erika.collipal@ufrontera.cl

Recibido: 12-05-2015

Aceptado: 22-07-2015 